Connection Machine to model the lateral eye of Limulus polyphemus and may, at this moment, be making video recordings in the Atlantic Ocean to use as visual input for his model.

The retina is able to adapt to complex temporal as well as spatial patterns of light ( $T$. Reuter, University of Helsinki). If an arbitrary light stimulus is repeated at constant intervals, most ganglion cells of the frog retina cease to produce a strong response. This is due to neither light adaptation nor spike adaptation, for slightly changing the frequency immediately restores the full response. $\mathrm{R}$. Desimone (NIMH, Bethesda) presented evidence that many neurons in inferior temporal cortex of macaques adapt to complex visual stimuli after a single exposure. If each stage of visual processing adapts out those aspects of the stimuli that it has analysed, only unexplained information reaches higher stages, as H. Barlow (University of Cambridge) has suggested. Knowing the right question to ask is often the key to progress, and Barlow provoked the audience by asking: "Why do you need your cerebral cortex?". At Oxford it was agreed that this was a very good question, but the most memorable answer was suggested to him by a Cambridge student: "You need your cerebral cortex so you know when to shut up". The answer may depend upon the species in rats, the cortex is not needed for the acquisition of conditioned fear, but is required to extinguish this response ( $\mathrm{J}$. LeDoux, New York University).

The output of the cerebral cortex is a good place to look for its behavioural significance, and eye movements are a favourable assay for visual function. S. Lisberger (University of California, San Francisco) and J. A. Movshon (New York University) have shown that the eye can track some moving targets coloured ones in particular - that are invisible to the classical 'colour-blind' motion pathway. Moreover the tracking system receives accurate signals about the speed of these targets even when the perceptual system incorrectly registers them as moving slowly. Neurons in the foveal region of the superior colliculus, an area of the midbrain known to be important for saccadic eye movements, have been discovered that discharge during active fixation of a visual target (R. Wurtz, NIMH); stimulation of these neurons delays saccades and lesions lead to an inability to suppress saccades, which occur with less than half the latency of normal saccades.

One way to go beyond the single neuron is to record from neural populations using voltage-sensitive dyes and intrinsic optical signals, and optical recordings have been used to map the spatial organization of neurons with similar properties, such as colour selectivity and disparity sensitivity, in area V2 of macaque visual cortex (D. Ts'o, Rockefeller University). Using the same techniques, A. Grinvald (Weizmann Institute, Israel) has made movies of cortical activity - probably subthreshold signals in dendrites - that resemble the sloshing of water in a swimming pool during an earthquake.

Widely separated pairs of neurons in the primary visual cortex of cats sometimes fire together at around $30-60 \mathrm{~Hz}$ (C. Gray, Salk Institute), and such synchrony has also been observed in other areas of cerebral cortex (M. Abeles, Hebrew University, Israel). These correlations are relatively rare, but a distributed population of coherently firing neurons could have much greater effect on target neurons because of temporal summation $^{6}$. It has been difficult to relate these observations to visual function, but they may teach us something about cortical circuitry ${ }^{7}$; for example models of inhibitory interactions between basket cells and cortical pyramidal neurons show that these inhibitory interneurons could help to synchronize local populations of neurons, and that under some conditions strong inhibition can actually speed up pyramidal neurons ${ }^{8}$.

Cognition is the holy grail of neuroscience. Visual attention is a window into our cognitive lives, a window that develops rapidly in infants, and it provides tags for objects as useful as any visual feature. Our short-term working memory gives us the ability to make plans and carry out complex actions, such as sequences of eye movements. Long-term memory allows us to form new habits and create new representations of the visual world. These connections between vision and behaviour were described by several speakers, and they point towards the emergence of a new world order without the traditional separation between vision, memory and motor systems. Having taken the brain to pieces, neuroscientists are now beginning the formidable task of putting it back together again.

Terrence J. Sejnowski is at the Howard Hughes Medical Institute in the Computational Neurobiology Laboratory, The Salk Institute, PO Box 85800 , San Diego, California 92138, USA.

1. Hubel, D. H. \& Wiesel, T. N. J. Physiol, Lond. 160 106-154 (1962)

2. Churchland, P. S. \& Sejnowski, T. J. Science 242 741-745 (1988)

3. Newsome, W. T., Britten, K. H. \& Movshon, J. A. Nature 341, 52-54 (1989)

4. Meister, M. Wong, R. O. L., Baylor, D. A. \& Shatz, C. J. Science 252, 939-943 (1991).

5. Mead, C. Analog VSLI and Neural System (AddisonWesley, Reading, Massachusetts, 1989).

6. Stryker, M. P. Nature 338, 297-298 (1989).

7. Bush, P. C. \& Douglas, R. J. Neural Computation 3. $19-30$ (1991).

8. Lytton, W. W. \& Sejnowski, T. J. J. Neurophysiol. (in the Lytton,
press).

\section{Electrons on tap}

HigH-temperature superconductors are already beginning to escape into technology. The electricity distribution industry would give them a particular welcome. Unfortunately, the problems of making, laying and joining up miles of brittle monolithic ceramic cable seem insuperable. Daedalus now has another approach.

He has been inspired by a recent report that an electron beam will thread its way safely through even a winding tunnel in a superconductor, by magnetic self-repulsion from the superconducting walls. An electron beam coasting in a vacuum is, of course, as lossless as the most superconducting cable. So Daedalus now plans to distribute electricity round the country in the form of electron beams in superconducting pipes.

The great advantage is that monolithic, electrically continuous superconductor is not needed. Lengths of superconducting pipe can simply be cemented together like traditional ceramic drain pipes or sewer conduits. The joints will have to be vacuum tight, of course, and the whole thing will need to be cooled in liquid nitrogen. Repelled from the walls of the pipe, the electron beam will then travel safely down the middle, undisturbed by the many joins and discontinuities. The pipe can confidently be laid round a curve: the beam will follow it. It can even be subdivided by $\mathrm{T}$-junctions and small offtakes, and controlled by standard taps and valves with superconducting gates or diaphragms. Electricity could be distributed in the same way as gas or water.

To cool a national distribution network in liquid nitrogen will call for a lot of new technology, but nothing that has not been thought about or tried already on a small scale. In a sense, electrical insulation will merely be replaced by thermal insulation. For a superconducting pipe repels electrons so perfectly that it never accumulates any charge itself. It can safely remain at earth potential.

DREADCO applications engineers are already wondering how best to use the electron beam when it arrives at the customer. The obvious approach, continuing this scale-up of vacuum-tube technology, is to let it hit a metal anode and be converted into a normal current. But electric light could be provided directly, by letting the beam hit a suitable phosphor, and television by scanning the beam over a screen. An electron-impact cooker could control the temperaturedistribution of a cake or roast with a precision unmatched by any microwave oven, while a simple electron leak could give a negative-ion generator for clearing the air and inducing a cheerful mental atmosphere.

David Jones 